\title{
Clinical and Radiological Study of Adenoid Cystic Carcinoma in Salivary gland, Sinonasal Tract and Nasopharyngeal
}

\author{
Bo-Hong SHI ${ }^{1, a, ~}$, Yong-Mei HUANG ${ }^{2}$, Tao QIN ${ }^{3}$ \\ 1Department of Stomatology, Rizhao People's Hospital, Rizhao, China \\ ${ }^{2}$ Department of Otolaryngology, Rizhao People's Hospital, Rizhao, China \\ ${ }^{3}$ Department of Radiology, Rizhao Port Hospital, Rizhao, China \\ aemail: shidoc@126.com \\ ${ }^{*}$ Corresponding author
}

Keywords: Adenoid Cystic Carcinoma, Salivary gland, Sinonasal, Nasopharyngeal, Clinical, Radiological, Diagnosis, Prognosis.

\begin{abstract}
Adenoid cystic carcinoma (ACC) is one of the most common primary malignancies of the major and minor salivary glands. ACC is an uncommon neoplasm that most frequently arises in salivary glands and related tissue in the head and neck region. Unlike head and neck squamous cell carcinoma, ACC is characterized by neural infiltration and abundant angiogenesis. The prognosis remains unsatisfactory, due to a high incidence of local recurrence and distant metastases that directly reduce the overall survival time. Primary salivary glands, sinonasal tract and nasopharyngeal ACC are uncommon tumors that are frequently misclassified, resulting in inappropriate clinical management. The purpose of this study was to to study the clinicopathological characteristics, imaging featuresand determine factors that impact recurrence of ACC. To improve the recognition and diagnosis of the disease, we conducted a retrospective review of 44 patients with salivary glands, sinonasal tract and nasopharyngeal ACC who were evaluated between 2010 and 2015. There were 23 men and 21 women were included in this study. CT and MRI was interpreted by experienced technologists, and the findings were reported by two experienced radiologists. The expressions of Staining for p63 and Ki-67 was performed. Our result shown ACC has distinct histologic features, with cribriform and tubular growth patterns of basaloid cells displaying a predominantly myoepithelial cellular phenotype. Middle or slightly low density on plain scan, mild or moderate enhancementon enhanced CT. Middle or slightly low signal on MRI T1WI sequence, middle or high signal on T2WI sequence, moderate to marked enhancement on enhanced T1WI middle or slightly low density on plain scan ,mild or moderate enhancementon enhanced CT. This neoplasm also had middle or slightly low signal on MRI T1WI sequence, middle or high signal on T2WI sequence, moderate to marked enhancement on enhanced T1WI. There were characteristics of common manifestations of malignant tumor including irregular and infiltratingmargin, cystoid or necrosis in the tumor, bony destruction, etc. This neoplasm also has uncommon clinical features of rare regional lymph node metastasis and a prolonged but relentlessly progressive clinical course. Clinical outcome in ACC is correlated to histologic grade. Perineural invasion was the most consistent predictors of poor outcome in head and neck ACC.
\end{abstract}




\section{Introduction}

Adenoid cystic carcinoma (ACC) is one of the most common primary malignancies of the major and minor salivary glands [1]. These are epithelial malignancies that develop at the expense of salivary glands, other rare localizations have been described in particular in the oral cavity, the sinonasal tract, lacrimal glands or nasopharynx. ACC is an uncommon neoplasm that most frequently arises in salivary glands and related tissue in the head and neck region. ACC is a relatively rare epithelial tumor characterized by neural and vessel invasion and a high incidence of distant metastasis [2]. Despite its slow growth, ACC exhibits high potential of recurrence. Clinical characteristics for ACC include invasive local growth, frequent local recurrence and distant metastasis. The conventional treatment for ACC is radical surgery with postoperative radiotherapy. Unlike head and neck squamous cell carcinoma, ACC is characterized by neural infiltration and abundant angiogenesis. Despite a relatively high short-term survival rate, the likelihood of recurrence and metastasis is high. Unlike head and neck squamous cell carcinoma, ACC is characterized by neural infiltration and abundant angiogenesis [2]. Most patients with ACC die due to a high incidence of distant metastasis and recurrence [3-5]. The prognosis remains unsatisfactory, due to a high incidence of local recurrence and distant metastases that directly reduce the overall survival time. Primary salivary glands, sinonasal tract and nasopharyngeal ACC are uncommon tumors that are frequently misclassified, resulting in inappropriate clinical management. The purpose of this study was to to study the clinicopathological characteristics, imaging featuresand determine factors that impact recurrence of ACC. To improve the recognition and diagnosis of the disease, we conducted a retrospective review of 44 patients with salivary glands, sinonasal tract and nasopharyngeal ACC who were evaluated between 2010 and 2015.

\section{Patients and Methods}

\section{Patients}

The present study was approved by the ethical committee at Rizhao People's Hospital. Written informed consent was obtained from all of the patients before their participation in the current study. The study was a retrospective study, and was conducted on 44 cases of primary salivary glands, sinonasal tract and nasopharyngeal ACC salivary glands, sinonasal tract and nasopharyngeal ACC were selected in the period from January 2010 to December 2015 at Rizhao people's Hospital, China, and a complete clinical and follow-up data were confirmed by surgery and pathology. There were 23 men and 21 women were included in this study. The patients aged 32-74 years old. The cases were retrieved from the files. CT and MRI was interpreted by experienced technologists, and the findings were reported by two experienced radiologists.

\section{Pathology Study}

Tissue samples were fixed in 10\% neutral buffered formalin and embedded in paraffin. Serial sections $(4 \mu \mathrm{m})$ were depar-affinized in xylene and hydrated through a graded series of ethanol. The pathological diagnosis was verified by histological methods independently by two pathologists, Tissue sections were deparaffinized and rehydrated using standard procedures. The pathological categorization was determined according to the current World Health Organization classification system and the pathological diagnosis was verified by histological methods independently by two pathologists. The 
pathological reading was determined for each slide with an overall pathological diagnosis determined for each subject. Immunohistochemical Ultra Sensitive ${ }^{T M} S-P$ method (Maixin-Bio, Fuzhou, Fujian, China) was used according to the manufacturer's instructions to detect differences in tumor tissue P63 and Ki-67 expression. Signals were visualized using the DAB substrate, which stains the target protein yellow. The positive controls were tissues known to express the antigen being studied. The negative controls were used. The primary antibody was replaced with PBS, containing $0.1 \%$ bovine serum albumin at the same concentration as the primary antibody.

\section{Statistical Analysis}

SPSS version 17.0 statistical software (SPSS Inc.: Chicago, IL, USA) was used to analyze the data. Enumeration data with $\chi^{2}$ test. The difference between the samples was considered significant when $P$ was less than 0.05 .

\section{Results}

44 patients were enrolled in this study included 23 men and 21 women with a median age of 51 years (range, 32-74 years). The patients presented clinically with a variety of symptoms referable to the tumor location where obstructive symptoms and epistaxis accounted for the most frequent presenting symptoms. Patients experienced epistaxis for a mean of 6.4 months, a finding which was not statistically significantly related to survival $(P>0.05)$. Patients frequently reported more than one symptom. 39 patients $(88.6 \%)$ had symptoms on presentation. 32 patients $(72.7 \%)$ presented with mass; 11 patients $(25.0 \%)$ presented with neurologic dysfunction, such as facial pain, numbness, or weakness; and the remaining 10 patients $(22.7 \%)$ presented with a range of symptoms that included nasal regurgitation, headache, congestion, vision changes, ear pain, hearing loss, hoarseness, and eye tearing. 11 patients $(25.0 \%)$ ACCs had lobular or infiltrating shape, whereas 2 with smooth margin, 3 patients $(6.8 \%)$ were originated from the lateral segmen to fexternal auditory canal with infiltrating margin, 31 patients $(71.5 \%)$ presented with middle or slightly low density on plain scan ,24 patients $(54.5 \%)$ presented with mild or moderate enhancementon enhanced CT, 10 patients $(22.7 \%)$ presented with middle or slightly low signal on MRI T1WI sequence ,10 patients $(22.7 \%)$ middle or high signal on T2WI sequence , moderate to marked enhancement on enhanced T1WI; 28 cases $(63.6 \%)$ had cystoid region in the tumor, shown in Fig 1 A-I. 4 patients $(9.1 \%)$ with destruction bony changes, $3(6.8 \%)$ cases with destruction and compression, and $4(9.1 \%)$ cases compression. ACCs present irregular and infiltratingmargin,cystoid or necrosis in the tumor, bony destruction, etc. Histologically, ACC are composed of small basaloid epithelial tumor cells, with small to moderate amounts of cytoplasm. The nuclei tend not to be pleomorphic, and have small or inconspicuous nucleoli. The tumor is composed of cells that exhibit either luminal epithelial differentiation or myoepithelial differentiation, with myoepithelial differentiation predominating 28 cases $(63.6 \%)$ presented with cribriform growth patterns, 24 patients $(54.5 \%)$ presented tubular growth patterns, 19 cases $(43.2 \%)$ presented with cribriform and tubular growth patterns, 4 (9.1\%) cases shown solid type of basaloid cells displaying a predominantly myoepithelial cellular phenotype. There was no statistical difference in mean age at presentation between the genders $(P>0.05)$. There was no significant difference in overall survival between the genders $(P>0.05)$. CD117 accentuated the epithelial cells, but tended to be found in areas in which a tubular histology predominated. The Ki-67 proliferation marker reacted with up to $20 \%$ of the nuclei, $41(93.2 \%)$ cases showed reaction in $<10 \%$, with an overall lack of a 
high proliferation index. Ki-67 and p53 expression was observed in $3(6.8 \%)$ and 2 $(4.5 \%)$ patients, respectively. There was no significant decrease in overall survival for patients who were $\geq 50$ years of age at initial presentation than those who were younger $(P>0.05$, respectively). Clinical outcome in ACC is correlated to histologic grade, perineural invasion, and the over expression of CD117 and Ki-67.
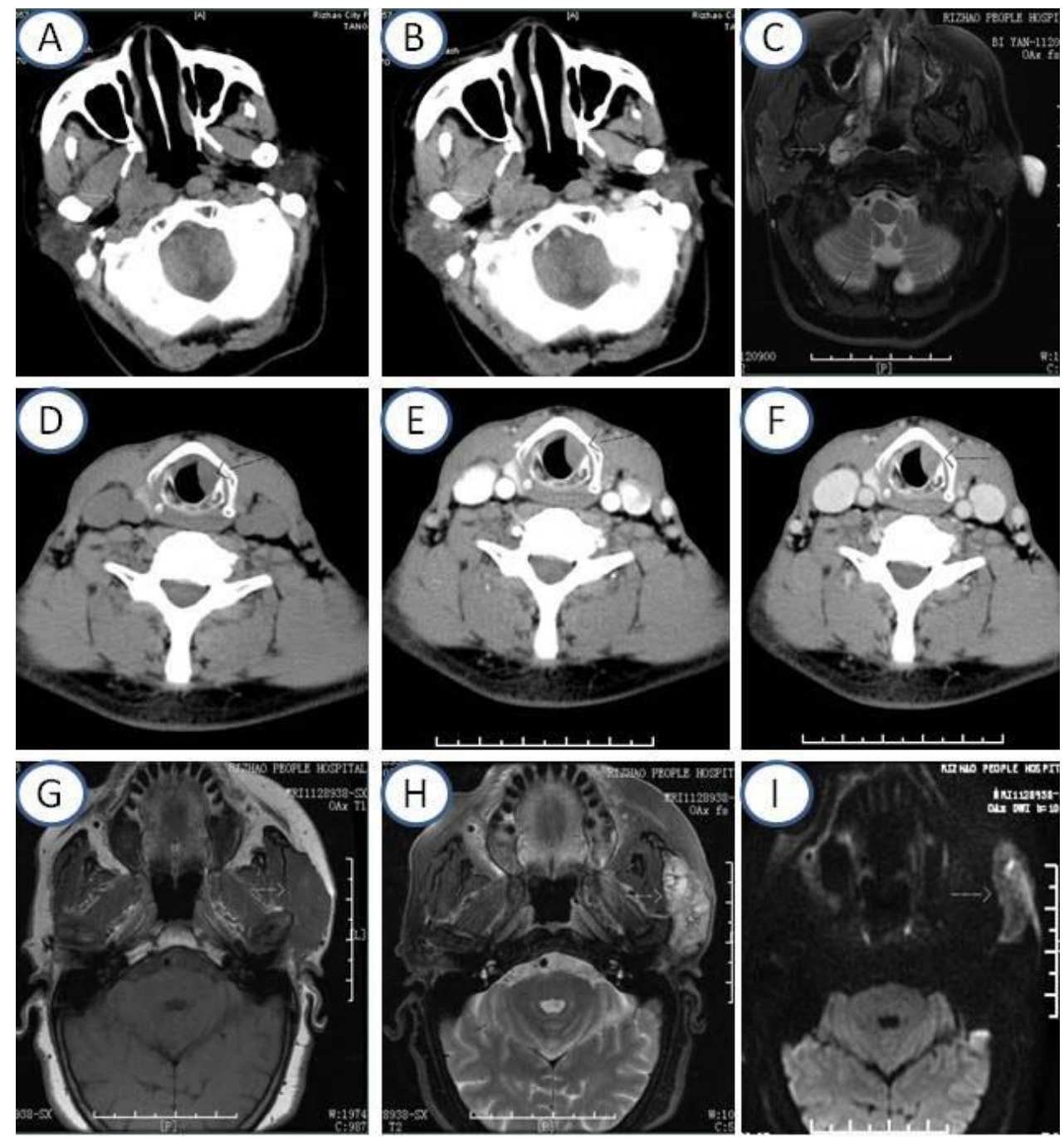

Figure 1. A-C shown nasopharynx Adenoid cystic carcinoma, computed tomography scan shown a mass with clear edges (A),moderate to marked enhancement on enhanced CT (B),MRI revealed a soft tissue marked enhancement on enhanced T1WI (C). D-F shown Left glottis lower area ACC, CT imaging revealed a mass with slightly low density on plain scan(D) and moderate enhancementon enhanced CT(E,F). G-I shown ACC in left parotid gland, MRI revealed a mass with middle signal T1 (G), high signal on T2WI sequence (H) on DWI (I).

\section{Discussion}

Adenoid cystic carcinoma (ACC) is the second most frequent salivary malignancy, accounting for $10-18 \%$ of all salivary malignancies $[1,6]$.The tumors is believed that ACC arises from the mucoserous glands of the upper aerodigestive tract. It occurred in the nasal cavity, nasopharynx, maxillary sinus, or in a mixed site such as nasal cavity, paranasal sinuses, including ethmoid, frontal, sphenoid, and/or nasopharynx. Imaging characteristic of adenoid cystic carcinoma is common manifestations of malignant tumor, including irregular and infiltratingmargin, cystoid or necrosis in the tumor,bony 
destruction, etc. The specific features are cribriform-cystoid sign, invading the nerve and infiltrating anatomic structure. The density and signal of adenoid cystic carcinoma is middle or slightly low density on pla in scan, mild or moderate enhancementon enhanced CT, middle or slightly low signal on MRI T1WI sequence, middle or high signal on T2WI sequence and moderate to marked enhancement on enhanced T1WI. Some adenoid cystic carcinoma have destruction or/and compression bony changes. ACC is characterized by a variety of histologic growth patterns, all of which may be exhibited in a single tumor mass. Histopathological grading of ACC is a controversial issue. It is generally agreed that solid type ACC has a relatively poor prognosis. However, the amount of solid regions within this often mixed type tumor that predicts a poor prognosis is not firmly established [6,7]. Some authors stipulate that the presence of a solid component regardless of the amount is a poor prognosticator where others argue that the amount should be taken into consideration. Several growth patterns have been described for ACC[8]. The most common and classic pattern is characterized by tumor cells arranged in variablysized nests of cells with distinct, punched-out spaces or pseudocysts, in a so-called cribriform pattern[5-9]. The cribriform spaces contain hyaline material, variably eosinophilic or basophilic in color. Most carcinomas with such an appearance will secrete mucin; however, in ACC, the secreted substances are basement membrane constituents, including proteoglycans [9]. A second growth pattern, typically mixed with the cribriform pattern, is a tubular pattern where the tumor infiltrates in separate gland-like groups with single central lumens. The third growth pattern is a solid growth pattern, where tumor cells grow in sheets without lumen formation. Clinical characteristics for ACC include invasive local growth, frequent local recurrence and distant metastasis. The histologic subtype of ACC has been suggested to be predictive of its biologic behavior, with the solid subtype being the most clinically aggressive. The histologic subtype of ACC has been suggested to be predictive of its biologic behavior, with the solid subtype being the most clinically aggressive. Our study indicate worse prognosis for the solid ACC subtype, it correlate with a higher proliferate index Ki-67. This may, however, be due to the low sample number with the solid pattern. Salivary gland ACC is a relatively rare epithelial tumor characterized by neural and vessel invasion and a high incidence of distant metastasis. Despite its slow growth, ACC exhibits high potential of recurrence. The conventional treatment for ACC is radical surgery with postoperative radiotherapy. Despite a relatively high short-term survival rate, the likelihood of recurrence and metastasis is high. Surgical resection followed by radiotherapy are suitable for treating the early stages of this malignancies in the absence of metastasis; chemotherapy is essential for management of advanced metastasis or local recurrence[10]. Surgical resection followed by radiotherapy is suitable for treating the early stages of this malignancies in the absence of metastasis; chemotherapy is essential for management of advanced metastasis or local recurrence. Further investigation of the possible biomarkers involved in the progression and metastasis of salivary ACC may help identify novel therapeutic strategies and improve the long-term outcome of the disease.Although combined imaging assessment has a definite detection benefit, this diagnostic method is applicable for screening purposes to women with unelevated risk of ACC, there are several limitations in our study. First, the cost of combined imaging assessment is not so attractive to patients. In addition, combined imaging assessment there would be some ionizing radiation in adition. 


\section{Conflict of Interest}

The authors declare no conflicts of interest.

\section{References}

[1] Kim KH, Sung MW, Chung PS, Rhee CS, Park CI, Kim WH. Adenoid cystic carcinoma of the head and neck. Arch Otolaryngol Head Neck Surg. 1994; $120: 721-726$.

[2] Ellington CL, Goodman M, Kono SA, Grist W, Wadsworth T, Chen AY, Owonikoko T, Ramalingam S, Shin DM, Khuri FR, Beitler JJ and Saba NF. Adenoid cystic carcinoma of the head and neck: Incidence and survival trends based on 1973-2007 Surveillance, Epidemiology, and End Results data. Cancer 2012; 118 : 4444- 4451.

[3] Moskaluk CA. Adenoid Cystic Carcinoma: Clinical and Molecular Features. Head and Neck Pathol.2013; 7:17-22.

[4] Hotte SJ, Winquist EW, Lamont E, MacKenzie M, Vokes E, Chen EX, Brown S, Pond GR, Murgo A and Siu LL. Imatinib mesylate in patients with adenoid cystic cancers of the salivary glands expressing c-kit: a Princess Margaret Hospital phase II consortium study. J Clin Oncol 2005; 23: 585-590.

[5] Papaspyrou G, Hoch S, Rinaldo A, Rodrigo JP, Takes RP, van Herpen C, Werner JA and Ferlito A. Chemotherapy and targeted therapy in adenoid cystic carcinoma of the head and neck: a review. Head Neck 2011; 33: 905-911.

[6] Wang Y-F, Ma S-R, Wang W-M, Huang C-F, Zhao Z-L, et al. (2014) Inhibition of Survivin Reduces HIF-1a, TGF-b1 and TFE3 in Salivary Adenoid Cystic Carcinoma. PLoS ONE 9(12):e114051. doi:10.1371/journal.pone.0114051

[7] Weerta SV, Waal IVD, Witte BI, Leemans CR, Bloemena E. Histopathological grading of adenoid cystic carcinoma of the head and neck: Analysis of currently used grading systems and proposal for a simplified grading scheme. Oral Oncology2015; 51:71-76.

[8] Szanto PA, Luna MA, Tortoledo ME, White RA. Histologic grading of adenoid cystic carcinoma of the salivary glands. Cancer. 1984;54:1062-1069.

[9] Franchi A, Santucci M, Wenig BM. Adenocarcinoma. In: Barnes EL, Eveson JW, Reichart P, Sidransky D, editors. Pathology and genetics head and neck tumours. Lyon: IARC Press; 2005.

[10] Chau NG, Hotte SJ, Chen EX, Chin SF, Turner S, Wang L and Siu LL. A phase II study of sunitinib in recurrent and/or metastatic adenoid cystic carcinoma (ACC) of the salivary glands: current progress and challenges in evaluating molecularly targeted agents in ACC. Ann Oncol 2012; 23: 1562-1570. 